\title{
Ocular complications in craniofacial fibrous dysplasia
}

\author{
G. M. LIAKOS, C. B. WALKER, AND J. A. S. CARRUTH \\ From the Southampton Eye Hospital and Southampton General Hospital
}

SUMMARY Three cases of craniofacial fibrous dysplasia are reported, all presenting to the ophthalmologist first. They all had ocular complications due either to direct involvement of the orbital bones or to secondary complications. Sphenoidal mucocele is added to the other complications previously reported, and accordingly a classification of the various ways that fibrous dysplasia can affect the ocular structures is proposed.

Fibrous dysplasia of the bone is a well documented disease that may affect any part of the skeletal system. The aetiology is unknown, but it is probably a developmental mesodermal disorder which may be triggered by trauma (Rees, 1976). The cavity of the affected bone is filled by fibrous tissue that may contain foci of calcified bone, hyalin, cartilage, cysts, and giant cells (Hutter et al., 1963). As a consequence there is diffuse thickening of the affected bones and encroachment on adjacent organs. The radiological appearance is usualty typical, but the importance of adequate biopsy is emphasised by the erroneous diagnosis in 25 of 90 reviewed cases by Harris et al. (1949). The differential diagnosis should include Paget's disease, osteopetrosis of Albers-Schönberg, and osteogenic sarcoma.

The disease is of particular interest to the ophthalmologist because it can affect the craniofacial bones either as a monostotic fibrous dysplasia or as a part of polyostotic fibrous dysplasia which is sometimes associated with skin pigmentation and endocrine disorders (Albright's syndrome). When the craniofacial bones are affected, encroachment on the paranasal sinuses, orbit, and foramina of the skull can produce visual loss, proptosis, diplopia, and epiphora (Moore, 1969), which more often than not are the first clinical manifestations of the disease.

In this paper we analyse the clinical and pathological findings in 3 cases of craniofacial dysplasia, all presenting to the ophthalmologist first, but each in a different way.

Correspondence to Dr G. M. Liakos, 44 Gordon Avenue, Winchester, Hants.

\section{Case reports}

CASE 1

A 12-year-old white boy presented to the Casualty Department of the Southampton Eye Hospital with the history of having fallen off his bicycle 1 week earlier. There was history of a similar blow on the head when the child was 5 years old, and since then he had a swelling on his left temple. There was no visual loss at any stage, and the general health was excellent.

Examination revealed the presence of left facial asymmetry with a bony swelling over the left frontal bone. The left globe was proptosed and displaced inferolaterally (Fig. 1). Visual acuity without correction in the right eye was $6 / 5$ and in the left $6 / 4$. Pupillary responses were normal in both eyes and fundus examination revealed no abnormality in either eye.

Skull $x$-ray films revealed a smooth, relatively

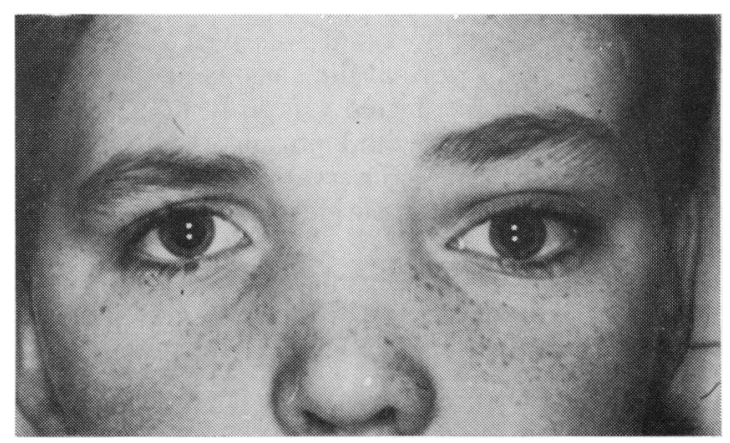

Fig. 1 Case 1. Note displacement of the left eye inferolaterally 


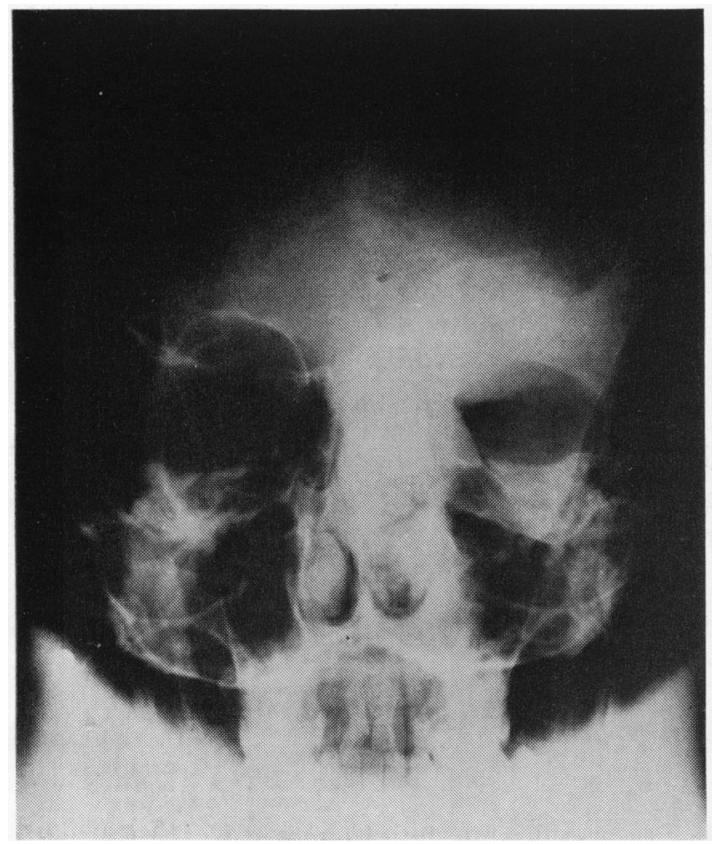

Fig. 2 Skull x-rays of case 1. Note thickening of bone on the left side, reducing the orbital size

homogeneous sclerosis and very considerable thickening of the bone on the left side (Fig. 2). This involved the roof of the orbit and extended forwards on to the frontal bone, all of the walls and roof of the maxillary antrum, and the wings of the sphenoid. It had resulted in obliteration of the frontal sinuses, the left ethmoids, and the sphenoid sinus. The left orbit was reduced in size by encroachment, as was the left side of the nasal cavity. The bony thickening also projected upwards into the anterior cranial fossa and the middle cranial fossa. As a result of the clinical and radiological findings a diagnosis of fibrous dysplasia was made.

Tomograms showed that in spite of the dense involvement of the lesser wing of the sphenoid on the left side the optic canal was well shown and appeared to be of normal dimensions.

In view of the unaffected vision, healthy appearance of the optic disc and normal dimensions of the optic canal, a conservative approach was adopted rather than a surgical decompression of the orbit.

Eleven years later, the appearance remains unchanged, with persisting proptosis but normal ophthalmoscopic and radiological findings.

\section{CASE 2}

A 31-year-old white man presented to the Casualty Department of the Southampton Eye Hospital complaining of a swelling over his left eye, present for 2 months and rapidly increasing in size. There was some history of trauma 22 years ago when he fell off a tree and suffered head injury. There were no visual complaints, and the general health was excellent.

Examination revealed some facial asymmetry, the bony structure being more prominent on the left side. There was a large mass in the superomedial quadrant of the left orbit displacing the globe inferolaterally (Fig. 3). Despite this there was no diplopia and the extraocular movements were full. Visual acuity without correction in the right eye was $6 / 5$ and in the left $6 / 6$. Fundus examination showed no abnormalities in either eye. Pupillary response to direct and consensual light was normal in both eyes.

Skull $x$-ray films revealed extensive involvement of the whole left side of the head, face, and jaws by fibrous dysplasia (Fig. 4). Every bone on that side

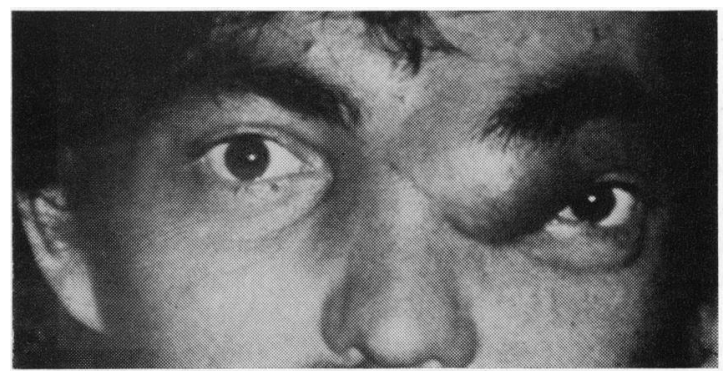

Fig. 3 Case 2. The left eye is displaced inferolaterally by a large mass in the superomedial quadrant

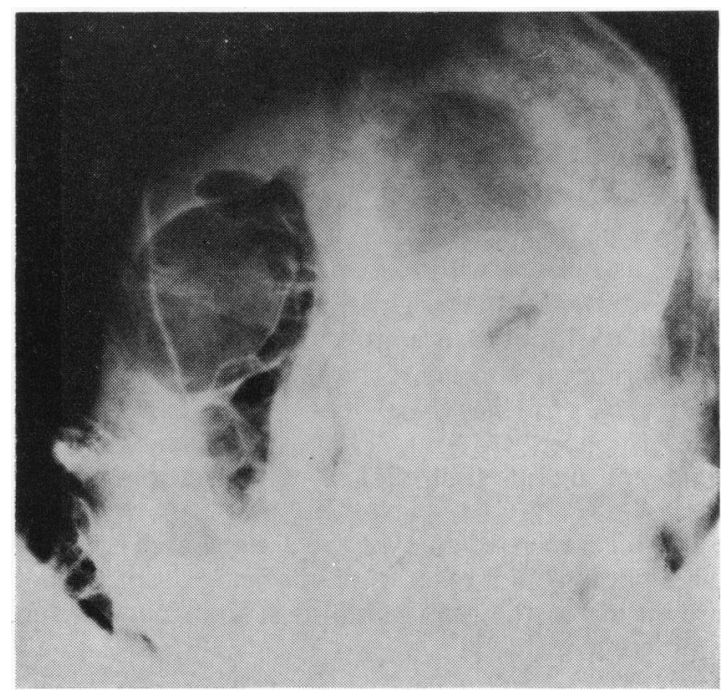

Fig. 4 Skull $\mathrm{x}$-rays of case 2. The anatomy of the left side is totally disrupted by remarkable thickening of the bones 


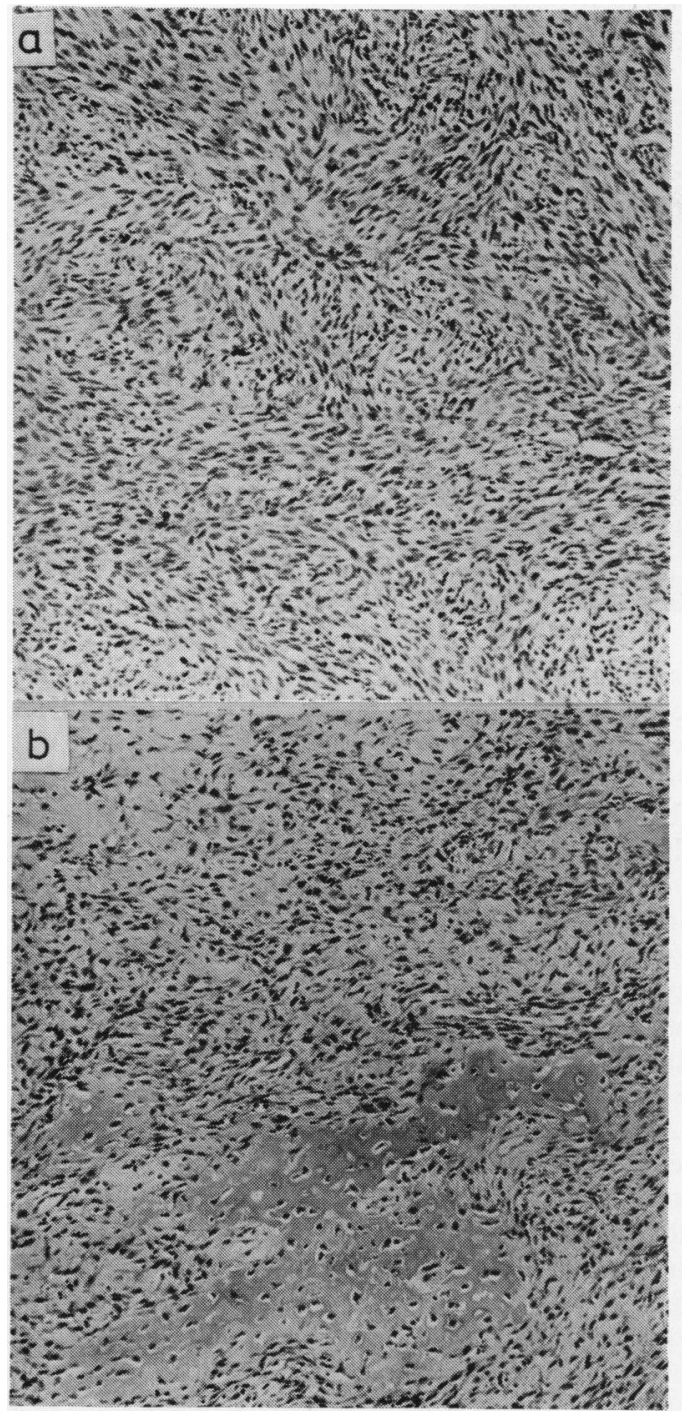

Fig. 5 Histology of the tumour in case 2. (a) Spindle cells arranged into storiform pattern $(H$ and $E, \times 80)$. (b) Note formation in parts of cartilage and osteoid $(H$ and $E, \times 80$ )

of the skull was involved, with remarkable thickening of the body of the sphenoid in particular. The superomedial portion of the left orbit was absent, and there was a $4 \mathrm{~mm}$ diameter well-defined lucency contiguous with the left orbit extending into the left frontal bone in the region of the frontal sinus. Chest $x$-ray films revealed fibrous dysplasia of the left 5 th, 6 th, and 7 th ribs.

An orbital tomogram confirmed the presence of an expansile destructive lesion involving the left frontal sinus. It was shown to have eroded the superomedial portion of the left orbit and to have opened into the left frontal fossa. The left maxillary antrum was diminutive, and there was enormous expansion and sclerosis of the sphenoid body and wings on the left side.

At this stage, it was thought that the orbital lesion was a frontal mucocele displacing the globe in the opposite direction, in spite of the rapidity of growth which is uncharacteristic of mucocele. It was only when an anterior orbitotomy was performed that the diagnosis of frontal mucocele proved to be erroneous.

A large nonencapsulated tumour was found instead, occupying the superomedial quadrant of the orbital cavity. The tumour was extending posteriorly and also superiorly invading and totally filling the frontal sinus. Laterally the tumour was free from attachments and was simply pushing the globe, but medially and superiorly it was infiltrating the orbital bones diffusely, hence making the removal difficult and incomplete.

Histological examination revealed the following: Macroscopically a white fleshy tumour $2 \times 3 \times 5$ $\mathrm{cm}$. Microscopically a spindle celled tumour, individual cells showing marked polymorphism. There were some individual tumour giant cells with plentiful cytoplasm. In part the spindle cells were arranged into storiform pattern. The mitotic rate was increased (Fig. 5a). Extra and decalcified section of the lesion showed that in parts the tumour cells were forming cartilage and osteoid (Fig. 5b).

\section{CASE 3}

A 20-year-old white woman presented to her local ophthalmologist complaining of rapid loss of vision in her left eye and partial loss of vision in her right eye, with normal fundi.

Skull $x$-ray films showed that the left ethmoidal air cells, in particular the posterior group, were dense. There was bulging and destruction of the orbital plate of the left sphenoid bone and of the nasal septum with obliteration of the left nasal air way. Further destruction extended into the basosphenoid across the full extent of the floor of the pituitary fossa. The destruction involved the left optic canal and the medial half of the right optic canal. There was considerable developmental asymmetry of the lesser wing of the sphenoid, and this, together with the bulging of the bone plates, suggested that the lesion may have been slowly growing before its more recent rapid advancement There was no evidence of soft tissue mass in the nasopharynx (Fig. 6).

When she was seen at the Neurosurgical Unit of the Southampton General Hospital, where she was 


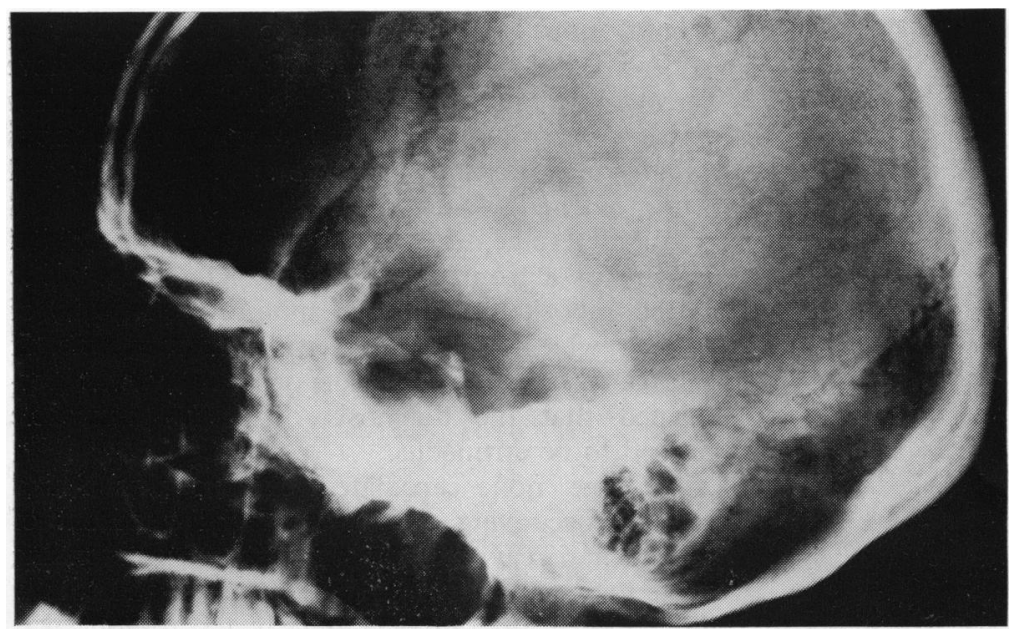

Fig. 6 Case 3. Destruction of the basosphenoid across the full extent of the floor of the pituitary fossa

referred, she was totally blind, having no perception of light in either eye. The left eye was slightly proptosed, but the extraocular movements were full. The pupillary reflexes were absent in both eyes, the pupils thus remaining fixed and dilated.

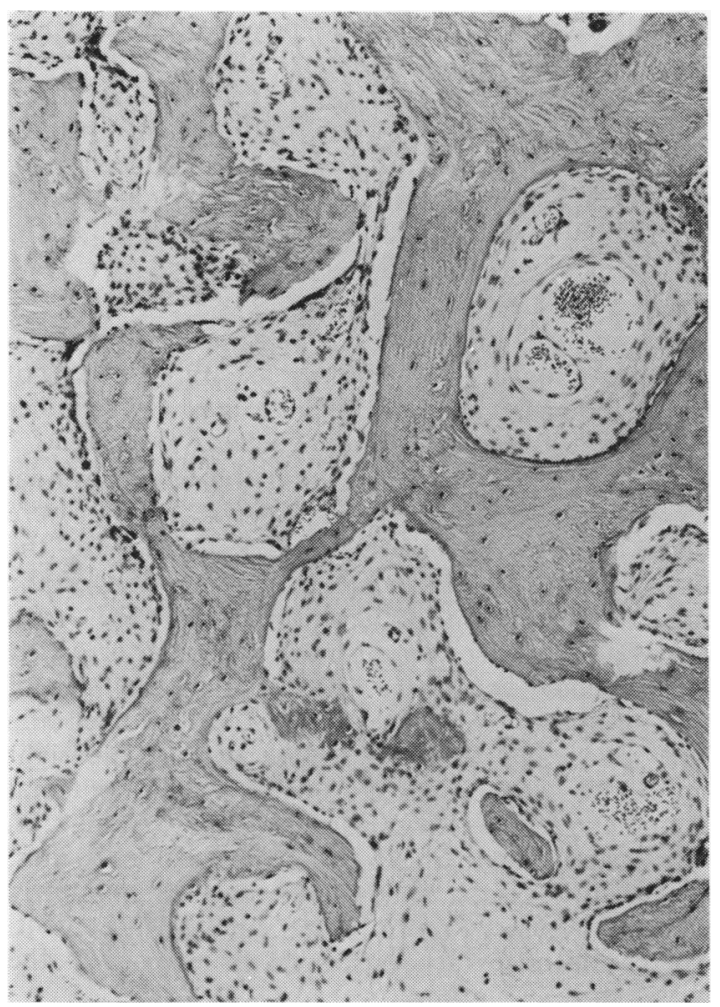

Fig. 7 Histology of fibrous dysplasia, with fibrous tissue invading the bone $(H$ and $E, \times 80$ )
Inspection of the fundus confirmed no abnormality. Neurological examination failed to demonstrate involvement of any other cranial nerves.

A CT scan confirmed the obliterations of the left ethmoid sinuses and destruction of the basosphenoid with bulging into the left orbit producing some proptosis and local deformity of the eyeball and deviating the left optic nerve.

At this point it was thought that the lesion was a tumour originating from the ethmoid sinuses and invading the left orbit. Accordingly treatment was introduced by means of local radiotherapy and systemic corticosterolds, but without improvement.

A bone biopsy was done next, which established the diagnosis of fibrous dysplasia (Fig. 7). Very soon after this, bifrontal craniotomy was performed, and confirmed the presence of fibrous dysplasia, mainly centred around the ethmoid and sphenoid sinuses. The most interesting finding, though, was a large mucocele originating from the sphenoid sinus and spreading out on both sides, encircling both optic nerves. Inside the cavity of the mucocele there was an extensive haemorrhage, obviously accounting for the acute visual loss.

Six months after the operation there was no return of visual function, and the discs were pale and atrophic. Skull $x$-rays revealed remarkable reconstitution of the pituitary fossa (Fig. 8).

\section{Discussion}

The first case illustrates the typical picture of proptosis due to fibrous dysplasia as described by other authors (Mortada, 1961; Moore, 1969; Gogi et al., 1976; Schonder, 1977; Friot et al., 1978). Every young patient with unilateral proptosis should be investigated for craniofacial fibrous 
Fig. 8 Postoperative appearance of case 3. Note remarkable reconstitution of the pituitary fossa and large frontal craniotomy

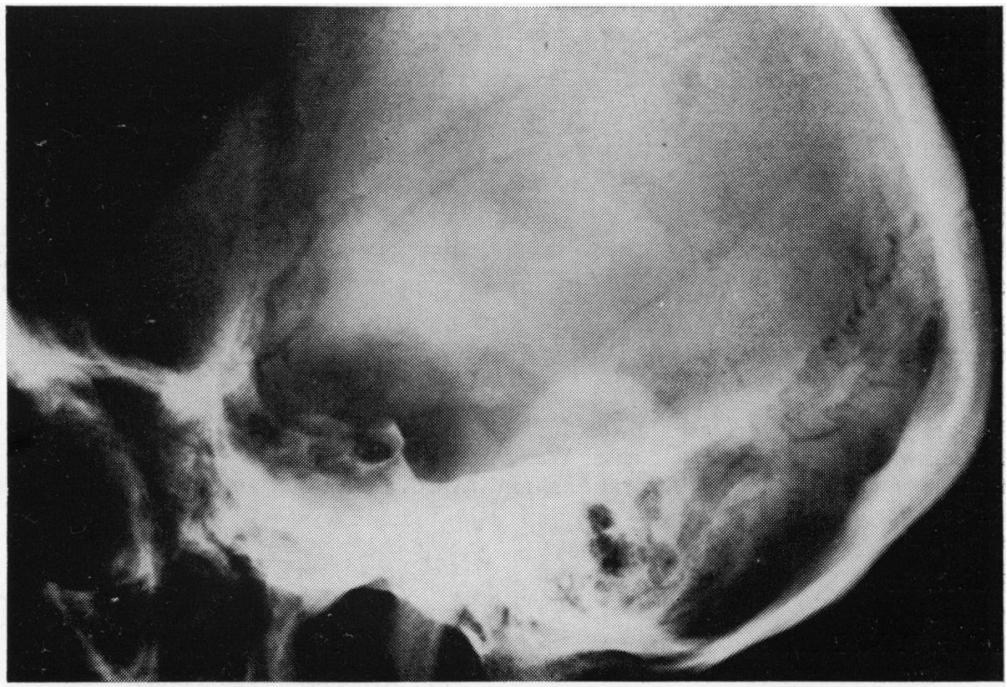

dysplasia (Daves et al., 1957; Natchiar, 1976). Radiological screening must include special views of the optic foramina, and surgical intervention is indicated when compression of the optic nerve becomes imminent. Unilateral involvement is the rule, but cases with bilateral visual impairment are found in the literature (Calderon et al., 1969; Sassis et al., 1968). Finney and Roberts (1976) described a case of fibrous dysplasia of the skull that developed trigeminal neuralgia, and we recently saw a case of 6th nerve palsy that was thought to be due to fibrous dysplasia. Thus it seems likely that besides the optic nerve other intracranial nerves can be involved as well.

The commonest complication of fibrous dysplasia is its malignant transformation to osteosarcoma (Coley et al., 1945; Jaffe, 1946; Perkinson and Higgenbotham, 1955; Yannapoulos et al., 1964; Schwartz and Alpert, 1964; Caron et al., 1971), and is demonstrated by the second case of this paper. The most interesting aspect of this case was the histology. Bony formation was scanty for a typical osteosarcoma, and a large amount of fibrous tissue gave the tumour a predominantly fibroblastic appearance. It is known that, histologically, osteosarcoma varies from case to case and from one area to another in the same tumour (Hogan and Zimmerman, 1962), but Jakobiek and Tannenbaum (1974) suggested that the fibroblast occupies a central position in the differentiation of many connective tissue tumours. If this theory is accepted, it is possible that many tumours reported as osteosarcomas because of some osteoid formation in fact arise from fibroblasts, some of which enter into morphological continua with the osteocyte. Two other rare forms of tumours complicating fibrous dysplasia have been reported on the basis of their histological appearance: adamantinoma (Baker et al., 1954) and cutaneous fibromyxoma (Lick and Viehweger, 1962).

The third case of this paper presents an interesting course of events combined with unusual clinical features and findings. Mucocele of the sphenoid sinus is rare (Nugent et al., 1970; McCarthy et al., 1972). Among the many neurological, nasal, and visual symptoms, gradual visual loss is sometimes the only clinical finding, as reported by Stanton (1970). The course is slowly progressive and may mimic pituitary neoplasm (Nevins and Leaver, 1967). Acute visual loss is uncharacteristic, and that renders our case unusual. But what makes our case unique is the presence of fibrous dysplasia, which can undoubtedly be regarded as the original pathology, the mucocele simply representing a complication. To our knowledge sphenoidal mucocele complicating fibrous dysplasia of the craniofacial bones has not been previously reported, although Grimaud et al. (1968), in a patient with fibrous dysplasia, described a mass invading the orbit from the frontal sinus, calling it ossifying fibroma, and Silva in the same year presented a similar lesion.

It is clear that fibrous dysplasia affects the ocular structures in many ways, and these are classified as follows:

A. Primary. (1) Frontal bone involvement resulting in proptosis, bony swelling. (2) Base of the skull involvement, resulting in extraocular muscle palsy and trigeminal neuralgia. (3) Optic canal involvement, resulting in visual loss and optic atrophy. 
(4) Body of the sphenoid involvement, resulting in chiasmal compression. (5) Maxillary bone involvement, resulting in epiphora.

B. Secondary. (1) Malignant transformation: (a) osteosarcoma, (b) fibrosarcoma, (c) adamantimoma, (d) cutaneous fibromyxoma. (2) Mucocele. (3) Ossifying fibroma.

We believe that this comprehensive list includes all the different ways that fibrous dysplasia can produce orbital or ocular symptoms, and can therefore be useful to the ophthalmologist.

We are grateful to Mr Jason Brice and Mr John Whitwell for allowing us to study their case. We should also like to thank Mr F. Sims for the illustrations, and Mrs J. Shapter for secretarial assistance.

\section{References}

Baker, P. L., Dockerty, M. B., and Coventry, M. B. (1954). Adamantimoma of the long bones. Journal of Bone and Joint Surgery, 36A, 704-720.

Calderon, M., and Brady, H. R. (1969). Fibrous dysplasia of bone. American Journal of Ophthalmology, 68, 513515.

Caron, A. S., Hajdu, S. I., and Strong, E. W. (1971). Osteogenic sarcoma of the facial and cranial bones. American Journal of Surgery, 122, 719-725.

Coley, B. L., and Stewart, F. W. (1945). Bone sarcoma in polyostotic fibrous dysplasia. Annals of Surgery, 121, 872-881.

Daves, M. L., and Yardley, J. H. (1957). Fibrous dysplasia of bone. American Journal of Medical Sciences, 234, 590-606.

Finney, H. L., and Roberts, T. S. (1976). Fibrous dysplasia of the skull with progressive cranial nerve involvement. Surgical Neurology, 6, 341-343.

Friot, J. M., Bleicher, B., Galoisy, C., Bretagne, M. C., Chobaut, J. C., and Wayoff, M. (1978). Four cases of exophthalmos of unusual naso-sinus origin. Revue d'otoneuro-ophtalmologie, 50, 141-149.

Gogi, R., Nath, K., and Krishna, G. (1976). Orbital fibrous dysplasia. Indian Journal of Ophthalmology, 24, 18-21.

Grimaud, R., Rauber, G., and Jeanin, C. (1968). Fibrous dysplasia of the frontal sinus. A new case. Revue d'otoneuro-ophtalmologie, 40, 165-168.

Harris, W., Dudley, H. R., and Barry, R. J. (1949). The natural history of fibrous dysplasia; an orthopaedic, pathological and roentgenographic study. Journal of Bone and Joint Surgery, 44, 207-233.

Hogan, M. J., and Zimmerman, L. E. (1962). Osteosarcomas: Ophthalmic Pathology, second edition, p. 753. Saunders: London.

Hutter, R. V. P., Foote, F. W., J. R., Frazell, E. L., and Francis, K. C. (1963). Giant cell tumours complicating Paget's disease of bone. Cancer, 16, 1044-1056.

Jaffe, H. L. (1946). Fibrous dysplasia of bone. Bulletin of New York Academy of Medicine, 22, 588-604.

Jakobiek, F. A., and Tannenbaum, M. (1974). The ultrastructure of orbital fibrosarcoma. American Journal of Ophthalmology, 77, 899-917.

Lick, R. F., and Viehweger, G. (1962). A contribution to the diagnosis of fibrous dysplasia of the skeletal system. Fortschritte auf dem Gebiete der Roentgenstrahlen und der Nuklearmedizin, 97, 33-38.

McCarthy, W. L., J. R., Frenkel, M., and Busse, B. (1972). Visual loss as the only symptom of sphenoid sinus mucocele. American Journal of Ophthalmology, 74, 1134-1140.

Moore, R. T. (1969). Fibrous dysplasia of the orbit. Survey of Ophthalmology, 13, 321-334.

Mortada, A. (1961). Fibrous dysplasia of the orbital bones. British Journal of Ophthalmology, 45, 737-740.

Natchiar, G. (1976). Unilateral proptosis due to fibrous dysplasia. Indian Journal of Ophthalmology, 24, 37-39.

Nevins, M. A., and Leaver, R. C. (1967). Sphenoid mucocele: an unusual mimic of pituitary neoplasm. Archives of Internal Medicine, 120, 607-611.

Nugent, G. R., Spinkle, P., and Bloor, B. M. (1970). Sphenoid sinus mucoceles. Journal of Neurosurgery, 32, 443-451.

Perkinson, N. G., and Higgenbotham, N. L. (1955). Osteogenic sarcoma arising in polyostotic fibrous dysplasia. Cancer, 8, 396-402.

Reese, A. B. (1976). Fibrous dysplasia of bone. Tumours of the eye, third edition, pp. 326-327. Harper and Row: London.

Sassis, J. F., and Rosenberg, R. N. (1968). Neurological complications of fibrous dysplasia of the skull. Archives of Neurology, 18, 363-369.

Schonder, A. (1977). Fibrous dysplasia of bone with proptosis. American Journal of Diseases of Children, 131, 678679.

Schwartz, D. T., and Alpert, M. (1964). The malignant transformation of fibrous dysplasia. American Journal of Medical Sciences, 247, 1-20.

Stanton, M. B. (1970). Sphenoid sinus mucocele. American Journal of Ophthalmology, 70, 991-994.

Yannapoulos, K., Bom, A. F., Griffiths, C. O., and Crikelair, G. F. (1964). Osteosarcoma arising in fibrous dysplasia of the facial bones; case report and review of the literature. American Journal of Surgery, 107, 556-564. 